\title{
Patient-controlled outpatient follow-up on demand for patients with rheumatoid arthritis: a 2-year randomized controlled trial
}

\author{
René Panduro Poggenborg ${ }^{1}$ (1) $\cdot$ Ole Rintek Madsen $^{1} \cdot$ Anne-Marie Tetsche Sweeney $^{1} \cdot$ Lene Dreyer $^{1,2}$. \\ Gunhild Bukh ${ }^{1}$. Annette Hansen ${ }^{1}$
}

Received: 24 August 2020 / Revised: 23 February 2021 / Accepted: 1 March 2021 / Published online: 6 March 2021

(C) International League of Associations for Rheumatology (ILAR) 2021, corrected publication 2022

\begin{abstract}
Introduction Scheduled routine visits in patients with rheumatoid arthritis (RA) may be in a stable period without active disease. Consequently, there is a demand for developing outpatient control procedures which cater to the needs of the individual patient. Objective This study aims to compare a patient-controlled outpatient follow-up system, Open Outpatient Clinic Programme (OOCP), with traditional scheduled routine follow-up (TSRF) regarding patient satisfaction and disease activity markers in RA patients.

Method In a 2-year randomized controlled trial, RA patients were allocated to OOCP or TSRF. OOCP patients had no scheduled appointments but were allowed acute appointments with their rheumatologist and had access to nurse-led consultations and a telephone helpline. Appointments for the TSRF group were scheduled according to routine procedures (clinical parameters: DAS-28, C-reactive protein, VAS pain, tender and swollen joint count, HAQ-DI and radiographs; psychological parameters: VAS patient satisfaction and EQ-5D).

Results Of 282 patients, 239 completed the study (OOCP/TSRF characteristics: age $61.4 \pm 10.5 / 60.9 \pm 12.2$ years, females $77 /$ $74 \%$, ACPA positive $66 / 65 \%$ ). At years 1 and 2 , OCCP had fewer visits (year 2: $2.6 \pm 1.6$ vs. $3.5 \pm 2 ; p<0.0005$ ) but more phone calls (year $2: 0.7 \pm 1.4$ vs. $0.1 \pm 0.3 ; p<0.0005$ ) compared to TSRF. OOCP was comparable to TSRF regarding clinical and psychological outcome measures, and no radiographic progression was observed.

Conclusions OOCP was associated with significantly fewer visits but with more phone calls to the nurse and was comparable with TSGentofte University HospitalRF regarding clinical, psychological and radiographic outcomes. Thus, the organization of outpatient care according to OOCP may be applied to strengthen patient-centred care in patients with RA.

ClinicalTrials.gov Identifier (July 20, 2020): NCT04476875

Key points

-In a patient-controlled outpatient follow-up system, RA patients had significantly fewer visits compared to traditional follow-up.

- The patient-controlled follow-up system was comparable with traditional follow-up regarding clinical, psychological and radiographic outcomes.

- Organization of outpatient care according to a patient-controlled follow-up system may be applied to strengthen patient-centred care in patients with $R A$.
\end{abstract}

Keywords Outpatient follow-up on demand $\cdot$ Rheumatoid arthritis

\section{Introduction}

Rheumatoid arthritis (RA) is a chronic inflammatory disease. Patients with RA suffer from fatigue, pain and swelling of

René Panduro Poggenborg poggenborg@dadlnet.dk

1 Center for Rheumatology and Spine Diseases, Copenhagen University Hospital Gentofte, Copenhagen, Denmark

2 Department of Rheumatology, Aalborg University Hospital, Aalborg, Denmark joints, and medical treatment and care are often lifelong [1]. In Denmark, the patients are traditionally closely monitored during the first year after diagnosis. During periods of stable disease, patients typically attend routine visits every 3-8 months at the rheumatology outpatient clinic. Arthritis may flare up between scheduled visits where it may be difficult to get acute appointments with the rheumatologist. Scheduled visits may be in a stable and 'good' period without active disease and any need for control or adjustment of treatment or care. The current coronavirus pandemic has emphasised the importance of reducing outpatient visits, when possible. Furthermore, it may be difficult to meet the patients' need 
for acute control when daily programmes are absorbed by routine controls. Studies have shown that it is important for patients to be able to control their disease. In particular, symptom management and emotional well-being are important factors for patients [2-4]. Thus, there is a demand for outpatient control procedures that cater to the needs of the individual patient and support the patient's experience of active participation in the control and treatment of their own disease. A British randomized controlled trial (RCT) study tested a direct-access system based on follow-up on demand, which showed that patients and doctors had increased satisfaction with direct access, and patients had $38 \%$ fewer control visits than those controlled at scheduled intervals [5]. Direct-access systems have been implemented at rheumatology outpatient clinics throughout the UK with positive results regarding disease control and patient satisfaction [6-8]. The successfulness of the system has not been tested in populations outside the UK.

The objective of the study was to compare an outpatient system for RA patients in Denmark based on patient selfcontrolled outpatient follow-up on demand, Open Outpatient Clinic Programme (OOCP), with traditional scheduled routine follow-up (TSRF) regarding patient satisfaction and effect on traditional disease markers.

\section{Material and methods}

\section{Design}

We performed a 2-year RCT with RA patients at the Center for Rheumatology and Spine Diseases, Copenhagen University Hospital Gentofte, capital region of Copenhagen, Denmark. At baseline, patients were randomly assigned (1:1) to the OOCP or TSRF group using a web-based secure centralised system with stratification according to biologic and non-biologic treatment [9]. Patients were evaluated at baseline and years 1 and 2, including assessment of disease activity and reporting of outcome measures on a touch screen at the clinic [10]. One blinded, senior project rheumatologist, who did not take part in the treatment or daily clinic, examined all the patients at baseline and years 1 and 2. The patient's usual rheumatologist performed the other visits, and in total, 13 rheumatologists in the clinic took part in the study.

\section{Recruitment}

The Danish National Patient Registry (DANBIO) was used to identify eligible patients with RA [9, 11]. DANBIO is an electronic register for monitoring clinical quality and has high nationwide coverage and completeness on data. At preplanned routine visits with the rheumatologist, identified patients were screened according to inclusion and exclusion criteria.

Patients with RA aged 18 to 80 years and with a disease duration of at least 1 year were included. If allocated to the intervention group, patients had to agree to take part in a short purpose developed patient education programme about RA, the intervention and how and when to contact the clinic (see Supplementary Appendix).

Patients who received monthly intravenous biological medicine, and thereby already closely monitored at the clinic, were excluded, as were patients who did not want to take part in patient education or felt they could not manage to make a decision about their disease.

\section{Access to treatment and care}

All patients were planned to have yearly scheduled project appointments with the study nurse and the study rheumatologist and to have outcome measures collected at baseline and years 1 and 2. Usual clinical standard procedures were followed regarding clinical examination, blood tests, radiographs and reporting of outcome measures on the electronic patient file system. The project visits were kept separate from the clinical treatment and care provided at the outpatient clinic. If any clinical issues were reported at the project visits, patients were referred to the outpatient clinic for follow-up with their contact rheumatologist and the healthcare team.

\section{Intervention}

The OOCP group had no scheduled appointments at the outpatient clinic, but they could book acute appointments with their contact rheumatologist within 5 days or less whenever they deemed it necessary. They also had access to nurse-led consultations without pre-booked appointments and to a nurse-led telephone helpline. Information and education on how to use the open outpatient system, the tested intervention, and how and when to contact the clinic were delivered by the study nurse when the patients were included in the study. Educational material had been prepared in cooperation with a patient partner from the Danish Rheumatism Association (https://www.gigtforeningen.dk/) and was used in all sessions to secure that the intervention patient received the same level and quality of information. Patients could ask questions and discuss issues they felt were important and were invited to contact the study nurse for more information or clarification. Topics were typically symptoms, challenges in RA and how to manage everyday life. The main message to the patients was to always call the clinic if in doubt. If there is any emergency, seek medical attention. The patients were informed to make sure to have their pre-booked standard blood tests taken every 8 weeks. They were assured that the results would be checked by a rheumatologist according to the 
standard clinical procedures and that they would be contacted by the clinic if there were changes that needed to be addressed.

The TSRF group had scheduled appointments every 3-8 months with their rheumatologist according to routine procedures at the outpatient clinic. They had access to acute appointments with their personal rheumatologist according to availability and to nurse-led consultations without prebooked appointments and access to a nurse-led telephone helpline. If there were no available appointment slots with the personal rheumatologist, they could be offered an appointment with another rheumatologist allocated to take acute appointments. They were informed to continue their standard routine programme at the clinic and to contact the clinic as usual if they had any clinical issues. If they had any questions regarding the study, they were invited to contact the study nurse for more information or clarification.

\section{Outcome measures}

Recorded clinical measures were Disease Activity Score (DAS-28) with C-reactive protein (CRP), the number of tender and swollen joints, visual analogue scale (VAS, 0-100) patient global assessment, VAS pain, VAS fatigue, Health Assessment Questionnaire Disability Index (HAQ-DI) and VAS physician global assessment. Recorded psychological measures were VAS patient satisfaction, VAS patient comfort, VAS patient involvement, Euro-Qol (EQ-5D) and VAS physician comfort and satisfaction (higher VAS indicates better outcome) [12], see Supplementary Appendix. The number of visits to the rheumatologist and phone calls to the nurse was registered in years 1 and 2. Radiographs of hands and feet were performed at baseline and years 1 and 2 .

\section{Statistics}

The Student's $t$ test was used to compare results from the OOCP and TSRF groups and the chi-squared test to compare binary outcomes. The last observation carried forward was assessed to handle missing data. A power calculation was performed. As the study focused on appropriate follow-up from a patient perspective, VAS patient satisfaction was selected for power analysis. It was estimated that the inclusion of 150 patients in the intervention group and 150 in the control group followed for 2 years would be required to detect a 0.24 difference between groups in VAS patient satisfaction, with $80 \%$ power at a $5 \%$ level of significance. Descriptive statistics with mean and standard deviation (SD) were used to characterize the patients. Cooperation with the DANBIO database was agreed upon, and a purpose-developed data program was set up within the database by ZiteLab ApS (https://zitelab.eu/) to accommodate the study. The statistical software package IBM SPSS Statistics v. 25 was used for the analyses.
Regulations for Good Clinical Practice were followed (https://www.ema.europa.eu/en/ich-e6-r2-good-clinicalpractice). The inclusion started in February 2015 and ended in January 2017. Data collection was concluded in December 2018, when the last included patients completed their year 2 project visits. Approval was granted by the National Scientific Ethical Committee (VEK Protocol No 38787-v2-19.12.2013) and the National Data Protection Agency. The study was performed in accordance with the ethical standards of the responsible committee on human experimentation (institutional and national) and with the Helsinki Declaration of 1975, as revised in 2000 and 2008 (https://www.wma.net/ what-we-do/medical-ethics/declaration-of-helsinki/dohoct2008/).

\section{Results}

Seven hundred and fifty RA patients were screened. We included 282 patients in the study, 266 patients completed the first year, and 239 the second year. Only a few patients $(18 / 25$ patients in the OOCP/TSRF group) did not complete the 2year study. Figure 1 shows the flow chart of the study participants. Baseline characteristics are shown in Table 1.

After 2 years, VAS patient satisfaction showed no statistically significant difference between the OOCP and TSRF groups ( $82 \pm 24$ vs. $83 \pm 23)$. No statistically significant difference in DAS-28 between the OOCP and TSRF groups was found ( $2.7 \pm 1.2$ vs. $2.5 \pm 1.0)$. Outcomes for OOCP at years 1 and 2 were comparable to TSRF regarding clinical and psychological outcome measures (Table 2; figures in Supplementary Appendix).

After 1 year, the OOCP group had $16 \%$ fewer visits than the TSRF group $(3.2 \pm 1.9$ vs. $3.8 \pm 1.6, p<0.05)$ and $31 \%$ fewer visits after 2 years $(2.6 \pm 1.6$ vs. $3.5 \pm 2.2, p<0.0005)$ (Table 2). Patients in the OCCP group made more telephone calls than the TSRF group $(1.8 \pm 3.3$ vs. $0.4 \pm 0.8, p<0.0005)$ after 1 year and after 2 years $(0.7 \pm 1.4$ vs. $0.1 \pm 0.3, p<$ $0.0005)$. Radiographic progression was seen in $2.9 \%(4 / 138)$ of the patients in the OOCP group and $2.1 \%$ (3/140) in the control group (non-significant). No adverse events were related to the follow-up strategy.

\section{Discussion}

We observed no differences in disease activity in patients following 2 years according to the open outpatient follow-up programme, OOCP, compared to patients receiving traditional routine follow-up, and no differences in radiographic progression were found. The intervention group had significantly fewer appointments but called the nurse more often than the 


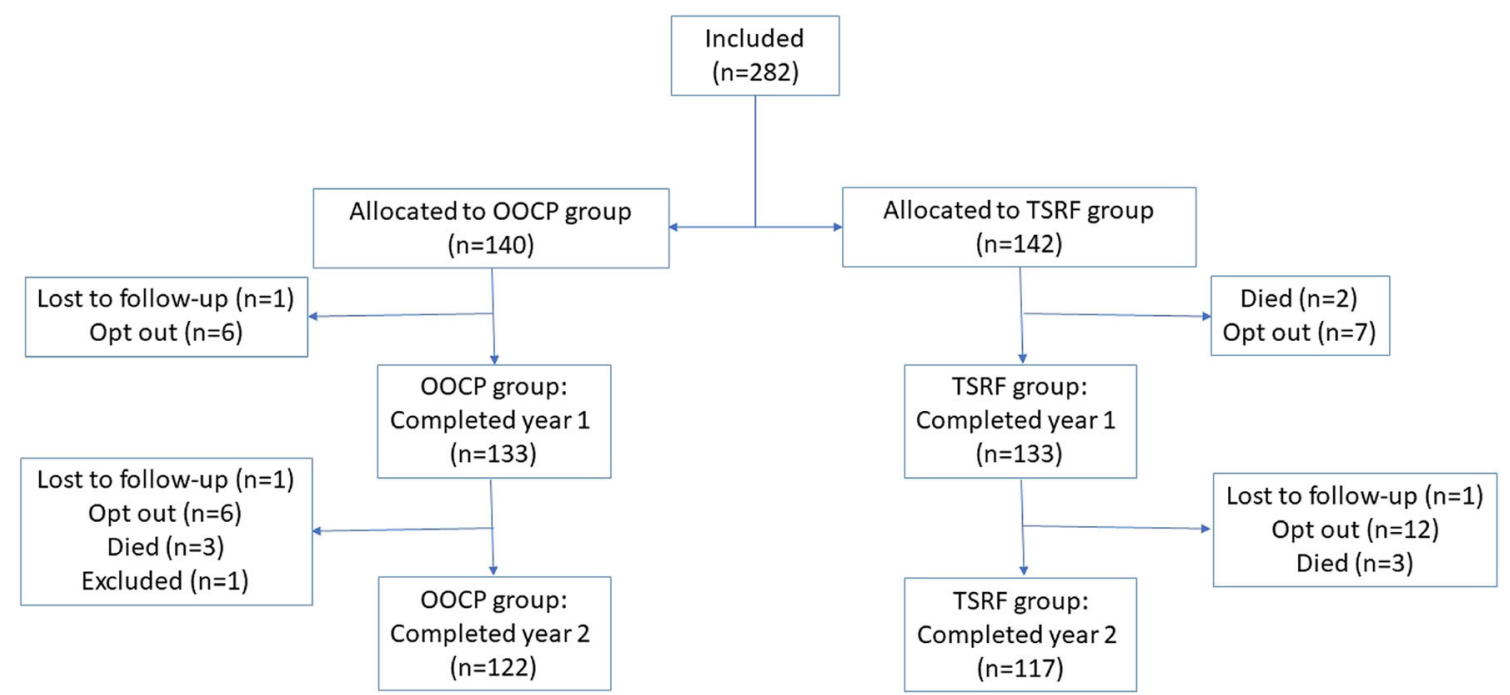

Fig. 1 Patient flow chart. Two hundred eighty-two rheumatoid arthritis patients were randomized to open outpatient clinic system (OOCP) or traditional scheduled routine follow-up (TSRF) in a rheumatology outpatient clinic in a 2-year follow-up study

control group. The groups were equally satisfied with and confident in their system of care.

The present study was inspired by a British RCT of 209 RA patients which found that a direct-access system based on follow-up on demand demonstrated increased patient satisfaction and fewer control visits than those controlled at scheduled intervals (5). In the British study, it was concluded that the patients found direct access more acceptable, associated with less overall use of healthcare resources, and the system was suggested to be tested in other hospital settings. As organizational differences exist between the British and Danish organizations of rheumatologic outpatient service, e.g. regarding the role of General Practitioners, we found it relevant to test the direct-access system in a Danish setting.

In the present study, the intervention group had access to acute appointments with their rheumatologist within 5 days, and both groups had open access to the nurse-led clinic without a pre-booked appointment and access to a nurse-led telephone helpline. This was a significant difference from the British study. In the British version, patients either followed the direct-access intervention or had no acute access to the outpatient clinic service other than by request from their general practitioner (5). The British patients could arrange an appointment with the rheumatologist with a maximum delay of 10 working days in the direct-access group patients.

We found that the two systems, OOCP and the standard clinical follow-up system with scheduled routine visits, proved equally effective regarding disease activity (DAS28) and level of patient satisfaction (0-100 VAS patient satisfaction 82 vs. 83 ). The British study had a more positive effect on patient satisfaction than found in our study (no significant change (0-10 VAS 8.9 to 9.3 ) in the directaccess group; decrease in the control group (0-10 VAS 8.9 to $8.3 ; p<0.02))(5)$. The difference is likely to be caused by the different set-ups of the two studies, e.g. in our study, acute appointments could easily be arranged relatively fast without involving the general practitioner.
Table 1 Baseline characteristics of the included 282 RA patients randomized to an open outpatient clinic system or traditional scheduled routine follow-up in a rheumatology outpatient clinic. Results are shown as mean $\pm \mathrm{SD}$ or percentages

\begin{tabular}{lll}
\hline & OOCP $(n=140)$ & TSRF $(n=142)$ \\
\hline Age (years) & $61.4 \pm 10.5$ & $60.9 \pm 12.2$ \\
Female (\%) & 77.1 & 73.9 \\
IgM-rheumatoid factor positive (\%) & 48.6 & 48.5 \\
ACPA positive (\%) & 66.4 & 64.9 \\
Erosive disease (\%) & 40.7 & 42.9 \\
Steroid treatment baseline (\%) & 10.0 & 7.7 \\
sDMARD treatment (\%) & 67.1 & 64.8 \\
bDMARD \pm sDMARD treatment (\%) & 32.9 & 35.2 \\
\hline
\end{tabular}

$A C P A$, anti-citrullinated protein antibody; $B D M A R D$, biologic disease modifying anti-rheumatic drug; $O O C P$, Open Outpatient Clinic Programme; $s D M A R D$, synthetic disease modifying anti-rheumatic drug; TSRF, traditional scheduled routine follow-up 
Table 2 Outcome measures in patients with RA randomized to Open Outpatient Clinic Programme or traditional scheduled routine follow-up in a rheumatology outpatient clinic at baseline and after 1 and 2 years of follow-up. Results are shown as mean \pm SD

\begin{tabular}{|c|c|c|c|c|c|c|}
\hline \multirow{2}{*}{$\begin{array}{l}\text { Time } \\
\text { Group }\end{array}$} & \multicolumn{2}{|l|}{ Baseline } & \multicolumn{2}{|l|}{ Year 1} & \multicolumn{2}{|l|}{ Year 2} \\
\hline & OOCP & TSRF & OOCP & TSRF & OOCP & TSRF \\
\hline VAS patient satisfaction $(0-100)$ & $88 \pm 21$ & $87 \pm 19$ & $84 \pm 25$ & $82 \pm 23$ & $82 \pm 24$ & $83 \pm 23$ \\
\hline No. of visits & & & $3.2 \pm 1.9$ & $3.8 \pm 1.6^{*}$ & $2.6 \pm 1.6$ & $3.5 \pm 2.2 * *$ \\
\hline No. of phone calls & & & $1.8 \pm 3.3$ & $0.4 \pm 0.8^{* *}$ & $0.7 \pm 1.4$ & $0.1 \pm 0.3 * *$ \\
\hline DAS-28 & $3.0 \pm 1.2$ & $2.9 \pm 1.0$ & $2.6 \pm 1.1$ & $2.6 \pm 1.0$ & $2.7 \pm 1.2$ & $2.5 \pm 1.0$ \\
\hline $\mathrm{CRP}(\mathrm{mg} / \mathrm{l})$ & $10.2 \pm 7.2$ & $10.1 \pm 8.0$ & $8.2 \pm 9.9$ & $5.7 \pm 5.1^{*}$ & $9.6 \pm 8.8$ & $5.5 \pm 8.9^{*}$ \\
\hline 28-SJC (0-28) & $0.6 \pm 1.5$ & $0.6 \pm 1.2$ & $0.2 \pm 0.5$ & $0.3 \pm 1.0$ & $0.3 \pm 0.9$ & $0.4 \pm 1.2$ \\
\hline 28-TJC (0-28) & $3.3 \pm 5.7$ & $2.4 \pm 4.2$ & $2.4 \pm 4.7$ & $2.1 \pm 3.7$ & $2.4 \pm 4.9$ & $2.3 \pm 4.7$ \\
\hline VAS pain $(0-100)$ & $27 \pm 25$ & $26 \pm 21$ & $28 \pm 26$ & $28 \pm 24$ & $32 \pm 27$ & $29 \pm 25$ \\
\hline VAS patient global $(0-100)$ & $33 \pm 28$ & $31 \pm 25$ & $33 \pm 27$ & $31 \pm 26$ & $32 \pm 25$ & $37 \pm 29$ \\
\hline VAS physician global (0-100) & $7.3 \pm 14$ & $5.5 \pm 10$ & $4.3 \pm 8.1$ & $5.1 \pm 10$ & $5.1 \pm 10$ & $6.3 \pm 13$ \\
\hline VAS fatigue $(0-100)$ & $40 \pm 27$ & $37 \pm 27$ & $41 \pm 28$ & $37 \pm 30$ & $44 \pm 29$ & $39 \pm 28$ \\
\hline HAQ-DI score $(0-3)$ & $0.6 \pm 0.6$ & $0.6 \pm 0.6$ & $0.7 \pm 0.6$ & $0.6 \pm 0.6$ & $0.8 \pm 0.7$ & $0.6 \pm 0.7$ \\
\hline EQ-5D & $0.8 \pm 0.2$ & $0.8 \pm 0.1$ & $0.8 \pm 0.2$ & $0.8 \pm 0.2$ & $0.8 \pm 0.2$ & $0.8 \pm 0.1$ \\
\hline VAS patient comfort $(0-100)$ & $90 \pm 16$ & $91 \pm 13$ & $88 \pm 18$ & $87 \pm 18$ & $85 \pm 21$ & $86 \pm 21$ \\
\hline VAS physician satisfaction $(0-100)$ & $95 \pm 13$ & $96 \pm 11$ & $95 \pm 1.8$ & $95 \pm 13$ & $96 \pm 12$ & $93 \pm 16$ \\
\hline VAS physician comfort (0-100) & $99 \pm 6.5$ & $99 \pm 5.7$ & $97 \pm 8.1$ & $96 \pm 11$ & $98 \pm 9.1$ & $95 \pm 14$ \\
\hline VAS patient inclusion $(0-100)$ & $90 \pm 17$ & $90 \pm 15$ & $86 \pm 21$ & $86 \pm 21$ & $83 \pm 22$ & $85 \pm 21$ \\
\hline
\end{tabular}

$* p<0.05 ; * p<0.0005$, OOCP vs. TSRF group (Student's $t$ test). DAS, Disease Activity Score; EQ-5D, European Quality of Life Index; HAQ-DI, Health Assessment Questionnaire Disability Index; No., number; OOCP, Open Outpatient Clinic Programme; pt, patient; SJC, swollen joint count; TJC, tender joint count; TSRF, traditional scheduled routine follow-up; VAS, visual analogue scale

Our findings are also supported by a previous Danish study of RA patients with low disease activity. In that study, a regime including shared care (rheumatologist and nurse) without planned consultations and a regime including only planned nursing consultations were both found safe to be implemented as a replacement of a traditionally planned rheumatologist consultation regime [13]. The shared care and nurse care regimen also seemed cost-effective [14].

Furthermore, our study results are in line with those from a Swedish study where $131 \mathrm{RA}$ patients were randomized to either patient-initiated appointments to a rheumatologist within 10 working days or to appointments in advance [15]. After 18 months, patient-initiated care was equal to traditional care in terms of RA outcomes. This approach was suggested for implementation to improve patient empowerment.

Recently, a systematic Cochrane review on patientinitiated appointment systems for adults with chronic conditions in secondary care was published [16]. Six broad health conditions were examined, including rheumatoid arthritis, chronic obstructive pulmonary disease and inflammatory bowel disease. The authors concluded that the certainty of the evidence was mainly low to very low, but little or no difference between patient-initiated and consultant-led appointment systems was found on quality of life or other aspects of disease status.
Considering the results of the current study, the OOCP could be offered as one of more follow-up options in RA at the rheumatology outpatient clinic. This has gained more importance in the time of the coronavirus pandemic. A study on the impact of the COVID-19 pandemic found that many patients with rheumatic and musculoskeletal diseases had hospital appointments cancelled or switched to telephone or video consultations [17]. A potential advantage of a patient-initiated access approach could be a reduction of missed hospital appointments, whereas increasing disease activity may not be discovered. More research is needed on the OOCP in multiple centres and in other inflammatory arthritis diseases to secure safety and quality. Out of 750 screened patients, 282 were included, and the reasons for nonparticipation were, e.g. the patients did not meet the inclusion criteria or declined to participate or the rheumatologist was not able to correctly complete the screening procedure. It is possible, that some patients declined to participate in the study as they were more comfortable with the current follow-up procedures and that others agreed to participate because of more positive attitudes to self-management. This may have resulted in a potential bias toward better outcomes in the intervention group. However, no significant differences in clinical and psychological outcomes were found between the OOCP and TSRF groups. In all circumstances, the open-access system should only be offered to motivated patients. 


\section{Conclusion}

In an RCT of RA patients, an open outpatient clinic system performed equally well as traditional scheduled routine procedures regarding clinical, radiographic and psychological outcomes after 1 and 2 years. The open programme was associated with significantly fewer visits but more phone calls to the nurse. A patient self-controlled follow-up on demand clinic system could provide an organizational supplement for selected patients with RA.

Supplementary Information The online version contains supplementary material available at https://doi.org/10.1007/s10067-021-05674-y.

Acknowledgements Niels Steen Krogh, ZiteLab ApS, is acknowledged for the continuous technical development and support of the DANBIO register.

Author contribution (1) All authors made substantial contributions to the conception or design of the work or the acquisition, analysis or interpretation of data; (2) drafting the work or revising it critically for important intellectual content; (3) approving the version to be submitted; and (4) agreeing to be accountable for all aspects of the work in ensuring that questions related to the accuracy or integrity of any part of the work are appropriately investigated and resolved.

Funding The study was solely funded by the Region the Municipality of Greater Copenhagen the Quality Fund (Region Hovedstaden, Kvalitetspuljen).

Data availability Data are available to the author group.

\section{Declarations}

Ethical approval Approval was granted by the National Scientific Ethical Committee (VEK Protocol No 38787-v2-19.12.2013) and the National Data Protection Agency.

Consent to participate Oral and written consents were acquired from all participants.

Consent for publication Oral and written consents were acquired from all participants.

Disclosures None.

\section{References}

1. Kristiansen TM, Primdahl J, Antoft R, Hørslev-Petersen K (2012) Everyday life with rheumatoid arthritis and implications for patient education and clinical practice: a focus group study. Musculoskeletal Care 10(1):29-38
2. Kristiansen TM, Primdahl J, Antoft R, Hørslev-Petersen K (2012) It means everything: continuing normality of everyday life for people with rheumatoid arthritis in early remission. Musculoskeletal Care 10(3):162-170

3. Cunha-Miranda L, Costa L, Ribeiro JS (2010) NEAR study: needs and expectations in rheumatoid arthritis - do we know our patients needs? Acta Reumatol Port 35(3):314-323

4. Van der Elst K, Meyfroidt S, De CD, De GA, Binnard E, Moons P et al (2016) Unraveling patient-preferred health and treatment outcomes in early rheumatoid arthritis: a longitudinal qualitative study. Arthritis Care Res (Hoboken) 68(9):1278-1287

5. Hewlett S, Kirwan J, Pollock J, Mitchell K, Hehir M, Blair PS, Memel D, Perry MG (2005) Patient initiated outpatient follow up in rheumatoid arthritis: six year randomised controlled trial. BMJ 330(7484): 171

6. Goodwin VA, Paudyal P, Perry MG, Day N, Hawton A, Gericke C, Ukoumunne OC, Byng R (2016) Implementing a patient-initiated review system for people with rheumatoid arthritis: a prospective, comparative service evaluation. J Eval Clin Pract 22(3):439-445

7. Whear R, Abdul-Rahman AK, Boddy K, Thompson-Coon J, Perry M, Stein K (2013) The clinical effectiveness of patient initiated clinics for patients with chronic or recurrent conditions managed in secondary care: a systematic review. PLoS One 8(10):e74774

8. Paudyal P, Perry M, Child S, Gericke CA (2012) Evaluation of a patient-initiated review system in rheumatoid arthritis: an implementation trial protocol. BMC Musculoskelet Disord 13:120

9. Hetland ML (2011) DANBIO - powerful research database and electronic patient record. Rheumatology (Oxford) 50(1):69-77

10. Secher AE, Glintborg B, Gudbergsen H, Krogh NS, Sorensen IJ, Jensen DV et al (2019) Comparing patient-reported outcomes entered at home versus at hospital, and testing touch screens for initial recruitment to scientific trials in arthritis patients. Scand J Rheumatol 48(3):178-184

11. Ibfelt EH, Jensen DV, Hetland ML (2016) The Danish nationwide clinical register for patients with rheumatoid arthritis: DANBIO. Clin Epidemiol 8:737-742

12. EuroQol Group (1990) EuroQol—a new facility for the measurement of health-related quality of life. Health Policy 16(3):199-208

13. Primdahl J, Sorensen J, Horn HC, Petersen R, Hørslev-Petersen K (2014) Shared care or nursing consultations as an alternative to rheumatologist follow-up for rheumatoid arthritis outpatients with low disease activity - patient outcomes from a 2-year, randomised controlled trial. Ann Rheum Dis 73(2):357-364

14. Sørensen J, Primdahl J, Horn HC, Hørslev-Petersen K (2015) Shared care or nurse consultations as an alternative to rheumatologist follow-up for rheumatoid arthritis (RA) outpatients with stable low disease-activity RA: cost-effectiveness based on a 2-year randomized trial. Scand J Rheumatol 44(1):13-21

15. Fredriksson C, Ebbevi D, Waldheim E, Lindblad S, Ernestam S (2016) Patient-initiated appointments compared with standard outpatient care for rheumatoid arthritis: a randomised controlled trial. RMD Open 2(1):e000184

16. Whear R, Thompson-Coon J, Rogers M, Abbott RA, Anderson L, Ukoumunne $\mathrm{O}$ et al (2020) Patient-initiated appointment systems for adults with chronic conditions in secondary care. Cochrane Database Syst Rev 4:CD010763

17. Michaud K, Wipfler K, Shaw Y, Simon TA, Cornish A, England BR, Ogdie A, Katz P (2020) Experiences of patients with rheumatic diseases in the United States during early days of the COVID-19 pandemic. ACR Open Rheumatol 2(6):335-343

Publisher's note Springer Nature remains neutral with regard to jurisdictional claims in published maps and institutional affiliations. 